\title{
Modeling of electrodes and implantable pulse generator cases for the analysis of implant tip heating under MR imaging
}

Article in Medical Physics · July 2015

DOI: $10.1118 / 1.4921019$

CITATIONS

2

3 authors, including:

\section{Volkan Acikel}

Bilkent University

25 PUBLICATIONS 18 CITATIONS

SEE PROFILE
READS

35

\section{Ergin Atalar}

Bilkent University

372 PUBLICATIONS $\quad 6,508$ CITATIONS

SEE PROFILE

Some of the authors of this publication are also working on these related projects: 


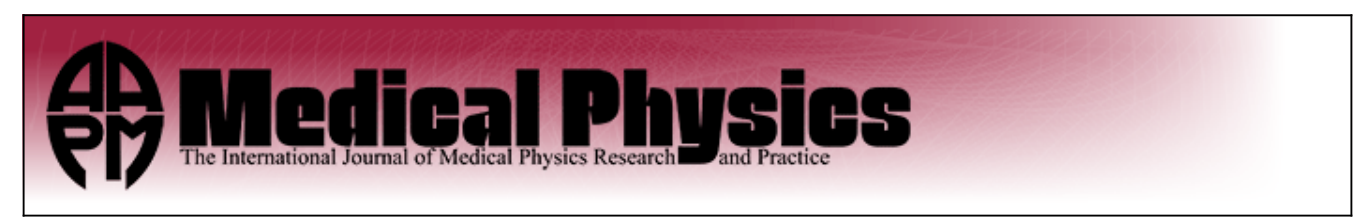

Modeling of electrodes and implantable pulse generator cases for the analysis of implant tip heating under MR imaging

Volkan Acikel, Ali Uslubas, and Ergin Atalar

Citation: Medical Physics 42, 3922 (2015); doi: 10.1118/1.4921019

View online: http://dx.doi.org/10.1118/1.4921019

View Table of Contents: http://scitation.aip.org/content/aapm/journal/medphys/42/7?ver=pdfcov

Published by the American Association of Physicists in Medicine

\section{Articles you may be interested in}

MR safety assessment of potential RF heating from cranial fixation plates at $7 \mathrm{~T}$

Med. Phys. 40, 042302 (2013); 10.1118/1.4795347

Dedicated phantom to study susceptibility artifacts caused by depth electrode in magnetic resonance imaging AIP Conf. Proc. 1494, 97 (2012); 10.1063/1.4764610

Designing passive MRI-safe implantable conducting leads with electrodes

Med. Phys. 37, 3828 (2010); 10.1118/1.3439590

Long term in vitro stability of fully integrated wireless neural interfaces based on Utah slant electrode array Appl. Phys. Lett. 96, 073702 (2010); 10.1063/1.3318251

MRI-compatible transurethral ultrasound system for the treatment of localized prostate cancer using rotational control

Med. Phys. 35, 1346 (2008); 10.1118/1.2841937

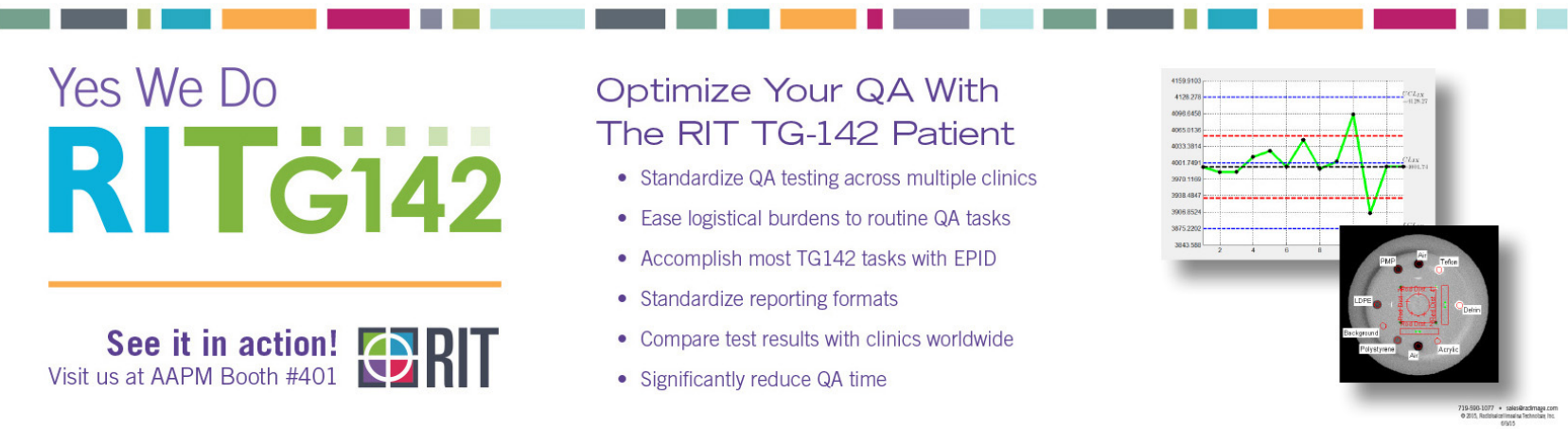




\title{
Modeling of electrodes and implantable pulse generator cases for the analysis of implant tip heating under MR imaging
}

\author{
Volkan Acikel ${ }^{\text {a) }}$ \\ Department of Electrical and Electronics Engineering, Bilkent University, Bilkent, Ankara 06800, Turkey \\ and National Magnetic Resonance Research Center (UMRAM), Bilkent, Ankara 06800, Turkey \\ Ali Uslubas ${ }^{\text {b) }}$ \\ MR:comp GmbH, MR Safety Testing Laboratory, Buschgrundstraße 33, 45984 Gelsenkirchen, Germany \\ Ergin Atalar \\ Department of Electrical and Electronics Engineering, Bilkent University, Bilkent, Ankara 06800, Turkey \\ and National Magnetic Resonance Research Center(UMRAM), Bilkent, Ankara 06800, Turkey
}

(Received 12 February 2014; revised 4 April 2015; accepted for publication 24 April 2015; published 10 June 2015)

Purpose: The authors' purpose is to model the case of an implantable pulse generator (IPG) and the electrode of an active implantable medical device using lumped circuit elements in order to analyze their effect on radio frequency induced tissue heating problem during a magnetic resonance imaging (MRI) examination.

Methods: In this study, IPG case and electrode are modeled with a voltage source and impedance. Values of these parameters are found using the modified transmission line method (MoTLiM) and the method of moments (MoM) simulations. Once the parameter values of an electrode/IPG case model are determined, they can be connected to any lead, and tip heating can be analyzed. To validate these models, both MoM simulations and MR experiments were used. The induced currents on the leads with the IPG case or electrode connections were solved using the proposed models and the MoTLiM. These results were compared with the MoM simulations. In addition, an electrode was connected to a lead via an inductor. The dissipated power on the electrode was calculated using the MoTLiM by changing the inductance and the results were compared with the specific absorption rate results that were obtained using MoM. Then, MRI experiments were conducted to test the IPG case and the electrode models. To test the IPG case, a bare lead was connected to the case and placed inside a uniform phantom. During a MRI scan, the temperature rise at the lead was measured by changing the lead length. The power at the lead tip for the same scenario was also calculated using the IPG case model and MoTLiM. Then, an electrode was connected to a lead via an inductor and placed inside a uniform phantom. During a MRI scan, the temperature rise at the electrode was measured by changing the inductance and compared with the dissipated power on the electrode resistance.

Results: The induced currents on leads with the IPG case or electrode connection were solved for using the combination of the MoTLiM and the proposed lumped circuit models. These results were compared with those from the MoM simulations. The mean square error was less than 9\%. During the MRI experiments, when the IPG case was introduced, the resonance lengths were calculated to have an error less than $13 \%$. Also the change in tip temperature rise at resonance lengths was predicted with less than $4 \%$ error. For the electrode experiments, the value of the matching impedance was predicted with an error less than $1 \%$.

Conclusions: Electrical models for the IPG case and electrode are suggested, and the method is proposed to determine the parameter values. The concept of matching of the electrode to the lead is clarified using the defined electrode impedance and the lead Thevenin impedance. The effect of the IPG case and electrode on tip heating can be predicted using the proposed theory. With these models, understanding the tissue heating due to the implants becomes easier. Also, these models are beneficial for implant safety testers and designers. Using these models, worst case conditions can be determined and the corresponding implant test experiments can be planned. (C) 2015 American Association of Physicists in Medicine. [http://dx.doi.org/10.1118/1.4921019]

Key words: magnetic resonance imaging (MRI), implant, safety, RF induced tissue heating

\section{INTRODUCTION}

Although magnetic resonance imaging (MRI) is a powerful diagnostic tool, patients who wear active implantable medical devices (AIMDs) cannot take advantage of MRI or can be scanned under restrictive conditions. In conventional MRI scanners, the radio frequency (RF) fields, which are essential for MRI, are generated by birdcage body coils. The coupling between AIMDs and the body coils may result in high E-field in the tissue around the AIMD, ${ }^{1}$ which can cause excessive 
tissue heating. ${ }^{2}$ The issues on the tip heating of the leads inside MRI scanners have been studied extensively. ${ }^{3,4}$ Progress has also been made in the MR safety of implants. ${ }^{5}$

Electromagnetic simulations and phantom experiments are the most powerful tools for the safety analysis of AIMDs. Although very accurate results can be obtained using these tools, they are valid only for the examined cases. To obtain a good understanding of the problem, the electromagnetic interaction between the AIMD which is under testing and the body coil must be simulated and tested by using MRI experiments under many different configurations. This process is very time consuming and very costly. A simple model of complete AIMD system including its electrode and case will be very beneficial for obtaining good intuition on the problem and also very helpful for designing more precise experiments. In our earlier study, the leads of the AIMDs were modeled using the modified transmission line method (MoTLiM). ${ }^{11}$ This model is not complete since it does not include the effects of the implantable pulse generator (IPG) cases and the electrodes. These, however, significantly change the induced currents on the leads and alter the implant heating problem. There are several studies in the literature for analyzing the effect of the IPG cases and electrodes on the implant heating. For example, Carmichael et al. ${ }^{6}$ investigated the issue on the tip heating of intracranial electroencephalograph (EEG) electrodes using two different tail configurations, i.e., open circuit and short circuit. However, another configuration may result in a worse case, which can be found by using the proposed method. Modeling of leads, electrodes, and IPG cases can prove helpful for understanding the interactions between them. Nordbeck et $a .^{7}$ analyzed 36 different cases by changing electrode and lead properties and showed the interactions between different leads and electrodes. Although these results illustrate the effect of electrodes and IPG cases on implant heating, a systematic method is required to better understand the behavior of these components of AIMDs.

Nitz et al. modeled the electrodes as resistive elements ${ }^{8}$ in their analysis of lead tip heating using DC measurements. However, the safety concern lies in the interaction of the implant with the RF pulses, and therefore, the analysis should be conducted at or around the Larmor frequency. A nonzero reactance in the equivalent circuit is expected in the electrode and the IPG case model. As will be shown in later parts of the paper, an additional voltage or current source is required to accurately model the electrode and the IPG case. The model parameters must be defined in relation to the RF scattering behaviors of the lead, the electrode, and the IPG case.
The effect of the implantable pulse generator on the tip temperature rise is rarely investigated in the literature. In the literature, there are studies that include IPG and show temperature rise for fully implanted systems. ${ }^{9,10}$ However, there are no clear data that show how IPG will change the temperature rise when it is introduced and removed.

In this paper, the IPG case and electrode parts of an AIMD are modeled such that they can be used in conjunction with the MoTLiM. ${ }^{11}$ The model of the IPG case and electrode are explained, following which the method used to find the model parameters is detailed. After finding the model parameters, induced currents on leads with the IPG case or electrode connections are determined using the MoTLiM. Finally, the proposed methods are tested using MRI experiments. During these analyses, bare cylindrical leads were used with a rectangular IPG case, a cylindrical electrode, and a spherical electrode. A lead is connected to an IPG case, and by changing the lead length, the temperature rise is measured and compared with the specific absorption rate (SAR) values, which are calculated using the IPG case model and the MoTLiM. Additionally, an electrode is connected to a lead via an inductor, and by changing the inductance, tip heating at the electrode is measured and compared with the SAR values, which are calculated using the circuit model.

\section{THEORY}

In our earlier study, we modeled the lead (not including its electrode or case) using a MoTLiM. ${ }^{11}$ In this model, the lead is assumed to be in a hypothetical shield. An hypothetical voltage between this shield and the lead and the current on the lead are formulated using well-known telegrapher equation. This model simplified the lead current calculation dramatically by replacing the lead with two parameters: the serial lead impedance per length and the equivalent wavenumber. The relationship between the scattering properties of the lead and environment was also demonstrated. In this study, as an extension of the previous study, electrode and IPG case of an AIMD are modeled, and using MoTLiM, their effect on lead tip heating is analyzed.

When a lossy body is considered, there are current loops inside the body due to the incident RF field. When a lead is placed inside the body, the patterns of these current loops change and follow a different loop, including the lead itself. Therefore, there is current flowing from the body to lead and from the lead to body along the lead. Figure 1 shows current

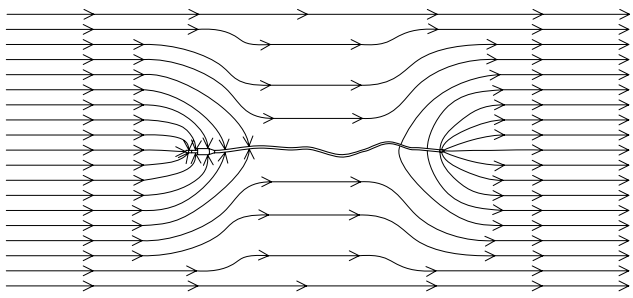

(a)

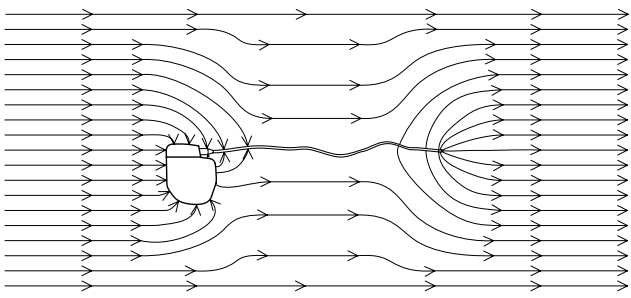

(b)

FIG. 1. (a) and (b) are sketch of the current patterns around a lead and a lead connected to a pulse generator, respectively. 
pattern inside the tissue in presence of a generic AIMD. In Fig. 1(a), the current flowing at the lead tissue boundary of the lead is shown without the IPG case. Because the lead is a source-free region, the total current flowing into and out from the lead must be zero. However, the presence of an IPG case or electrode changes these currents along the lead. In Fig. 1(b), the current pattern at the boundary of a lead and the body is shown for a lead connected to an IPG case. It can be observed that total current at the lead and tissue boundary is not zero. However, in this case, there must be a source. To show this effect for the IPG case and electrode, a current source is used in the circuit model. Since the magnitudes of conduction and displacement currents are comparable, a complex impedance is used in order to model the interaction between the IPG case and the electrode with the tissue. Therefore, the circuit model of the electrodes and IPG cases has a current source $\left(I_{c}\right)$ and impedance $\left(Z_{c}\right)$, as shown in Fig. 2(a). However, to be consistent with the previous studies in the literature ${ }^{8,12}$ and because boundary conditions are easily defined throughout the paper, instead of a current source and impedance, a Thevenin equivalent $\left(V_{c}\right.$ and $\left.Z_{c}\right)$ is used.

After defining the circuit models, the scattering problem can be converted into a simple circuit problem and then solved. This circuit model is easy to use with previously published model, which is based on the modified transmission line circuit model $^{11}$ as shown in Fig. 2(b). $Z$ is the series impedance, and $Y$ is the shunt admittances of the lead. From the circuit model of the lead, wavenumber along the lead was defined as $k_{t}=\sqrt{-Z Y}$.

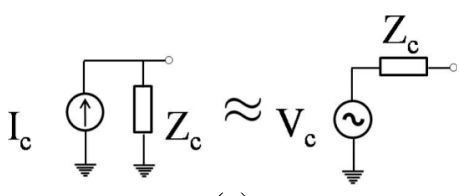

(a)

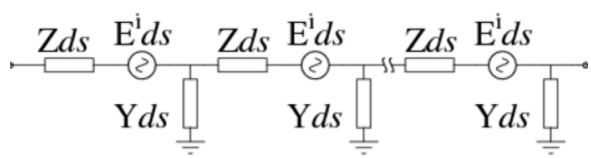

(b)

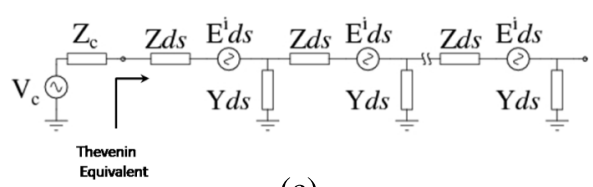

(c)

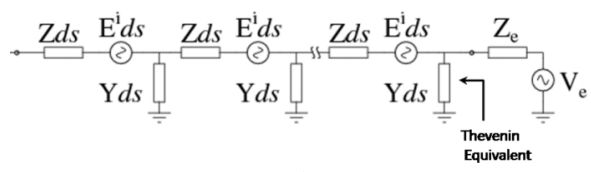

(d)

FIG. 2. (a) The IPG case and electrode model as the current source and impedance and the Thevenin equivalent circuit. (b) The modified transmission line model. Distributed voltage sources were introduced to show the effect of the incident field. (c) The IPG case model connected to the modified transmission line model. (d) The electrode model connected to the modified transmission line model.
In the MoTLiM, ${ }^{11}$ the behavior of the induced currents was modeled using the following second order differential equation:

$$
I(s)+\frac{1}{k_{t}^{2}} \frac{d^{2} I(s)}{d s^{2}}=\frac{E^{i}(s)}{Z},
$$

where $k_{t}=\sqrt{-Z Y}$ is the wavenumber along the lead, $Z$ is the distributed impedance, $s$ is the position along the lead, $I(s)$ is the current on the lead, and $E^{i}(s)$ is the tangential component of the incident E-field. Equation (1) was derived from the modified lumped element model of the lead. Using the same model, an equation for the hypothetical voltage, which is useful for defining some boundary conditions, can be derived as

$$
V(s)=\frac{Z}{k_{t}^{2}} \frac{d I(s)}{d s} .
$$

Using the equation of continuity, it can be observed that the hypothetical voltage is the scaled version of the charge distribution along the lead. However, using the hypothetical voltage can help define associated concepts throughout the paper. For the sake of simplicity, the proposed theory is explained using uniform E-field exposure. However, the concepts that are explained throughout the paper can be rederived for any known incident E-field.

\section{A. Modeling of the IPG case and electrode}

To explain the method, we consider an IPG case and determine its parameter values. In MoTLiM, leads were modeled as shown in Fig. 2(b) with series impedances, $Z$, and shunt admittances, $Y$, for infinitesimally small portions of lead. First, the Thevenin equivalent of a lead is found at the point shown in Fig. 2(c) using the MoTLiM. Solving Eqs. (1) and (2) with a uniform E-field, the current and hypothetical voltage along the lead can be found as follows:

$$
\begin{aligned}
& I(s)=A e^{-j k_{t} s}+B e^{j k_{t} s}+\frac{E_{i}}{Z}, \\
& V(s)=\frac{-j Z}{k_{t}}\left(A e^{-j k_{t} s}-B e^{j k_{t} s}\right),
\end{aligned}
$$

where $E_{i}$ is the tangential component of the uniform incident field, and $A$ and $B$ are the unknowns which will be found using boundary conditions.

Here, the Thevenin equivalent of the lead, with length $l_{m}$, from the terminal at $s=-l_{m} / 2$ will be found when the other end of the lead $\left(s=l_{m} / 2\right)$ is floating inside the tissue. To find the open circuit voltage, $V_{o c}$, the boundary conditions in Eq. (4) can be applied to Eq. (3) and the unknowns $A$ and $B$ are thus determined,

$$
\begin{aligned}
I\left(s=-l_{m} / 2\right) & =0, \\
I\left(s=l_{m} / 2\right) & =0 .
\end{aligned}
$$

Since the end of the lead at $s=l_{m} / 2$ is floating inside the tissue, the current will be zero. Please note that this condition merely states that there will not be any current flowing from the wire to the places other than the surrounding medium. The current flowing to the medium is possible, and in fact, it is 
typically very high around the tip of the wire. At $s=-l_{m} / 2$, to find the $V_{o c}$, current must be zero. After replacing the $A$ and $B$ in Eq. (3), $V_{o c}$ is found as follows:

$$
V_{o c}=\frac{E_{i}}{k_{t}} \tan \left(k_{t} l_{m} / 2\right) \text {. }
$$

To find the short circuit current, $I_{s c}$, the boundary conditions in Eq. (6) are applied to Eq. (3), and the unknowns $A$ and $B$ are determined,

$$
\begin{array}{r}
V\left(s=-l_{m} / 2\right)=0, \\
I\left(s=l_{m} / 2\right)=0 .
\end{array}
$$

After replacing $A$ and $B$ in Eq. (3), $I_{s c}$ is found as follows:

$$
I_{s c}\left(s=-l_{m} / 2\right)=\frac{E_{i}}{Z}\left(1-\frac{1}{\cos \left(k_{t} l_{m}\right)}\right),
$$

where $l_{m}$ is the lead length. $V_{o c}$ is the Thevenin voltage, and the Thevenin impedance can be found as follows:

$$
Z_{\mathrm{th}}=-\frac{Z}{k_{t}} \cot \left(k_{t} l_{m}\right) \text {. }
$$

Using the Thevenin equivalent of the lead and the IPG case model, Kirchhoff's voltage equation can be written as follows:

$$
V_{c}-I\left(s=-l_{m} / 2\right) Z_{c}-I\left(s=-l_{m} / 2\right) Z_{\mathrm{th}}-V_{\mathrm{th}}=0,
$$

where $I$ is the current at the connection point of the IPG case and the lead. However, in Eq. (9), there are three unknowns. $I$ can be found using method of moments (MoM) simulations, but there is still a need for a second equation to find the remaining two unknowns. Therefore, a second lead, for example, with different lengths, can be used for these calculations, and a second Kirchhoff's voltage equation is obtained. Then, solving the Kirchhoff's voltage equations for two leads simultaneously, the parameters of the IPG case can be found.

To find the parameters of the electrode circuit model $\left(V_{e}\right.$ and $Z_{e}$ ), the same method can be applied.

\section{B. Induced currents on lead with electrode and IPG case}

Because the birdcage coils are widely used as transmit coils in MRI and they have a fairly uniform E-field distribution along their $z$-axis, uniform E-field exposure to the implants is not an unlikely situation. Therefore, we consider a lead under uniform E-field incidence with length $l_{m}$. We connect the lead to an IPG case at position $s=-l_{m} / 2$ and leave the other end of the lead at position $s=l_{m} / 2$ floating inside the medium.

Solving Eqs. (1) and (2) with a uniform E-field, the current and hypothetical voltage along the lead are found, as in Eq. (3). To find the unknowns $A$ and $B$, the following boundary conditions are applied:

$$
\begin{aligned}
V\left(s=-l_{m} / 2\right)-I\left(s=-l_{m} / 2\right) Z_{c}-V_{c} & =0, \\
I\left(s=l_{m} / 2\right) & =0,
\end{aligned}
$$

where $V_{c}$ is the IPG case voltage and $Z_{c}$ is the IPG case impedance. The same boundary condition can be used for the electrode by replacing $V_{c}$ with the electrode voltage $\left(V_{e}\right)$ and $Z_{c}$ with the electrode impedance $\left(Z_{e}\right)$. Applying the boundary conditions in Eq. (10), the following equation system with two unknowns $A$ and $B$ can be found:

$$
\begin{aligned}
& \frac{-j Z}{k_{t}}\left(A e^{j k_{t} l_{m} / 2}-B e^{-j k_{t} l_{m} / 2}\right) \\
& \quad-\left(A e^{j k_{t} l_{m} / 2}+B e^{-j k_{t} l_{m} / 2}+\frac{E_{i}}{Z}\right) Z_{c}-V_{c}=0, \\
& A e^{-j k_{t} l_{m} / 2}+B e^{j k_{t} l_{m} / 2}+\frac{E_{i}}{Z}=0 .
\end{aligned}
$$

Solving Eq. (11), the unknowns $A$ and $B$ and, consequently, the induced current and the hypothetical voltage can be found.

\section{C. Calculation of the rise in tip temperature}

Circuit models of the IPG case and electrode need to be verified; however, they are not directly measurable quantities. Therefore, for the verification of the circuit models, their effect on the rise in tip temperature at the lead tip is used. Instead of calculating the rise in tip temperature directly, the square of the hypothetical voltage is used. The hypothetical voltage is the scaled version of the charge on the lead. Assuming that the scattered fields decay fast due to the conductivity of the tissue, the square of the hypothetical voltage has a linear relationship with the SAR and rise in temperature at the lead tip.

\section{C.1. Formulation of the rise in tip temperature at the lead tip and effect of the IPG case}

When a uniform E-field excitation, $E^{i}(s)=E_{0}$, is applied, the current and hypothetical voltage along the lead can be found as in Eq. (3). Let the lead be located between $s=-l_{m} / 2$ and $s=l_{m} / 2$. If both ends of the lead are not connected to any IPG case or electrode, the current at $s= \pm l_{m} / 2$ will be zero. Therefore, the induced current and the hypothetical voltage along the lead can be found as follows:

$$
\begin{aligned}
& I(s)=\frac{E_{0}}{Z}-2 \frac{E_{0}}{Z} \frac{\sin \left(k_{t} \frac{l_{w}}{2}\right)}{\sin \left(k_{t} l_{w}\right)} \cos \left(k_{t} s\right), \\
& V(s)=2 \frac{E_{0}}{k_{t}} \frac{\sin \left(k_{t} \frac{l_{w}}{2}\right)}{\sin \left(k_{t} l_{w}\right)} \sin \left(k_{t} s\right) .
\end{aligned}
$$

Then, at the lead tip, the square of the hypothetical voltage becomes

$$
|V|^{2}=\left|\frac{E_{0}}{k_{t}} \tan \left(k_{t} l_{w} / 2\right)\right|^{2} .
$$

Assuming that the scattered fields are decaying fast due to the loss of the surrounding medium, the hypothetical voltage has an approximate quadratic relationship with the rise in temperature at the tip of the lead $\left(s=l_{m} / 2\right)$ and can be formulated as follows:

$$
\Delta T \approx C\left|\frac{E_{0}}{k_{t}} \tan \left(k_{t} l_{w} / 2\right)\right|^{2},
$$

where $C$ is a constant, which depends on thermal properties of the medium as explained in Yeung et al., ${ }^{2}$ and also includes the spatial averaging effect. ${ }^{13}$ In this work, the value of $C$ was not calculated, and therefore, the above equation was considered as a proportionality. 
To see the effect of the IPG case, a lead with length $l_{m}$ was connected to the IPG case at $s=-l_{m} / 2$, and the square of the hypothetical voltage was calculated at $s=l_{m} / 2$. Under uniform E-field exposure, the hypothetical voltage distribution along the lead was found using Eq. (3). Additionally, the unknowns $A$ and $B$ must be found by applying the following boundary conditions:

$$
\begin{aligned}
I\left(l_{m} / 2\right) & =0, \\
V\left(s=-l_{m} / 2\right)-Z_{c} I\left(s=-l_{m} / 2\right)-V_{c} & =0,
\end{aligned}
$$

where $Z_{c}$ and $V_{c}$ are the case impedance and voltage, respectively. By determining the unknowns $A$ and $B$, the square of the hypothetical voltage can be found.

\section{C.2. Effect of matching the lead impedance to the electrode impedance}

The electrode impedance indicates the interaction of the electrode with the tissue. Therefore, the dissipated power associated with the real part of the electrode impedance is the power dissipated in the tissue. From this point of view, it can be considered the scaled version of the SAR around the electrode. Therefore, the dissipated power associated with the real part of the electrode has a linear relationship with the rise in temperature. First, the Thevenin equivalent of the lead is found, as in Eqs. (5) and (8) from the point shown in Fig. 2(d). Then, using the Thevenin equivalent and the circuit model of the electrode, the dissipated power from the electrode can be approximated as follows:

$$
\operatorname{Re}\left\{P_{e}\right\}=R_{e}\left(\frac{V_{\mathrm{th}}-V_{e}}{Z_{\mathrm{th}}+Z e}\right)\left(\frac{V_{\mathrm{th}}-V_{e}}{Z_{\mathrm{th}}+Z e}\right)^{*}
$$

where $R_{e}$ is the real part of the electrode impedance. If a matching impedance is placed between the electrode and the lead, as shown in Fig. 3, the dissipated power on the electrode can be calculated as follows:

$$
\operatorname{Re}\left\{P_{e}\right\}=R_{e}\left(\frac{V_{\mathrm{th}}-V_{e}}{Z_{\mathrm{th}}+Z e+Z_{m}}\right)\left(\frac{V_{\mathrm{th}}-V_{e}}{Z_{\mathrm{th}}+Z e+Z_{m}}\right)^{*} .
$$

Although the perfect power matching condition cannot be achieved with a single element, the imaginary part of the sum of the Thevenin impedance of the lead and the electrode impedance can be canceled out. Under this condition, the dissipated power from the electrode can be maximized. Any change in the value of the matching impedance will reduce the dissipated power.

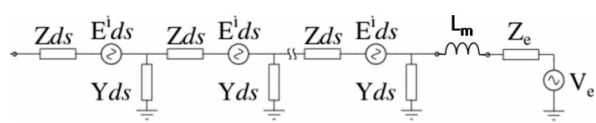

(a)

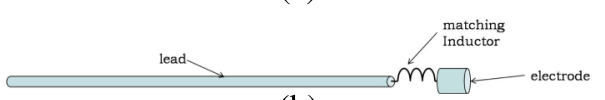

(b)

Fig. 3. (a) Modified transmission line model connected to the electrode via a matching inductor. (b) Connection of the lead to the electrode via a matching inductor.

\section{METHOD}

The circuit model parameters of the IPG case and electrode were found using the MoTLiM and MoM together. Then, their validity was checked using the MoM simulations and MRI experiments. For the MoM simulations, FEKO was used (EM Software \& Systems Germany, Böbligen, GMbH), and MRI experiments were conducted using the $3 \mathrm{~T}$ Siemens TimTrio system.

\section{A. IPG case circuit model}

The circuit model of the IPG case was tested using MoM simulations and MRI experiments. A rectangular box with dimensions $1.2 \times 4.4 \times 5 \mathrm{~cm}$, which are close to commercially available pulse generator dimensions, was used for both the simulations and experiments. For the simulations, the box was considered to be a perfect electric conductor (PEC). For the MRI experiments, a copper box was built; however, the loss of copper was ignored for the calculations. A circular phantom was prepared for the experiment and filled with HEC solution. The relative permittivity and conductivity of the phantom material were measured as 55 and $0.17 \mathrm{~S} / \mathrm{m}$, respectively, at $123 \mathrm{MHz}$. Phantom gel was prepared such that its electrical properties are close to electrical properties of human muscle tissue. $^{14}$

To find the values of IPG case circuit model parameters, the method explained in Sec. 2.A was used. Bare wires with a diameter of $0.5 \mathrm{~mm}$ and lengths of 15 and $45 \mathrm{~cm}$ were connected to the PEC box. The current at the connection point of the IPG case and wire was found using the MoM for both wire lengths. To validate the model parameter, a bare wire with a diameter of $1.3 \mathrm{~mm}$ was connected to the PEC box, and the induced current under uniform E-field on the leads was solved for lead lengths of $40.3,35.3$, and $27.3 \mathrm{~cm}$, which are chosen to show that models are capable of solving the oscillatory behavior of the induced currents. The results were compared with the MoM simulations. During MoM simulations, a lead was placed on $z$-axis and the PEC box was connected to it. PEC box was meshed with maximum edge length of $0.5 \mathrm{~mm}$ and leads were segmented with maximum segment length of $2 \mathrm{~mm}$. All geometry was illuminated by four plane electromagnetic waves propagating in $x,-x, y$, and $-y$ directions and $\mathrm{E}$ field component was in $z$ direction at $123 \mathrm{MHz}$ to emulate the birdcage coil. The IPG case model was also tested using MRI experiments. The IPG case was connected to the bare lead with a diameter $1.3 \mathrm{~mm}$ and positioned inside the phantom, as shown in Fig. 4(a). This configuration ensures that the lead is exposed to a uniform E-field. The length of the lead was changed from 5.3 to $45.4 \mathrm{~cm}$, and the temperature rise at the lead tip was measured using a fiber optic temperature sensor (Optical Temperature Sensor, Neoptix Reflex-4 RFX134A) as shown in Fig. 4(b). The square of the hypothetical voltage at the lead tip was calculated and compared with the experimental data. Using the same lead and removing the IPG case, the experiments to measure the rise in the tip temperature were repeated by changing the lead length from 11 to $55 \mathrm{~cm}$. Lead lengths were chosen to show the shift in resonance peaks. 


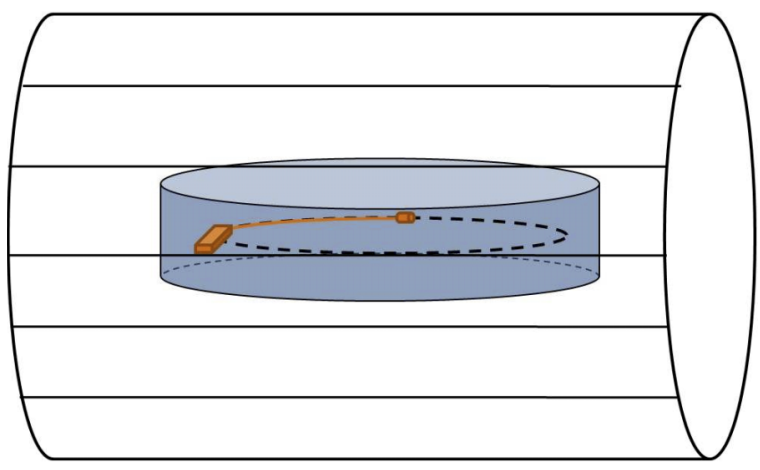

(a)

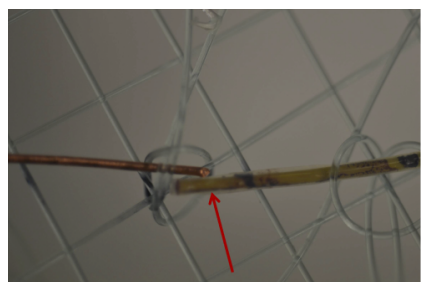

(b)

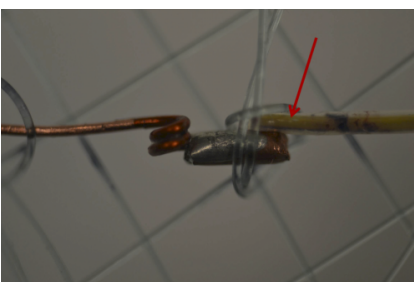

(c)
FIG. 4. (a) is the experimental setup. In all of the experiments, with the IPG case or the electrode, leads were placed on a circular path as shown, with the black circle inside the cylindrical phantom. The phantom was placed inside the MR scanner to be cocentered with the transmit body birdcage coil. (b) and (c) Placement of the temperature sensors with respect to the lead and electrode, red arrows show the fiberoptic temperature sensors. In (c) an inductor can be seen between the lead and electrode. Setup is placed on a net formed of fishing line and fixed using knots.

\section{B. Electrode circuit model}

The circuit model of the electrode was also tested using both MoM simulations and MRI experiments. For the simulations, a spherical electrode with a $1 \mathrm{~mm}$ radius was used, and the circuit parameters inside the medium were determined to be as follows: conductivity of $0.42 \mathrm{~S} / \mathrm{m}$ and relative permittivity of 81 , which are close to electrical properties of brain tissue at $123 \mathrm{MHz} .{ }^{14}$ The electrode was connected to bare leads with a radius of $0.5 \mathrm{~mm}$ and lengths 10 and $30 \mathrm{~cm}$. During MoM simulations, leads were positioned along the $z$-axis, and a spherical electrode was connected to them. The electrode was meshed with maximum edge length of $0.5 \mathrm{~mm}$ and the leads were segmented with maximum segment length of $2 \mathrm{~mm}$. The geometry was excited with plane electromagnetic wave similar to the previous simulations. The induced currents at the connection points of the leads to the electrode were found using MoM simulations for both lead lengths. Then, the Thevenin equivalents of both the leads were found using MoTLiM, and the values of the circuit model parameters were found. Then, the spherical electrode was connected to bare leads with a radius of $0.1 \mathrm{~mm}$ and lengths 25,35 , and $45 \mathrm{~cm}$. Induced currents on the leads were solved for uniform E-field incidence using the MoTLiM, and the results were compared with the MoM simulations. For the MRI experiments, using an inductor between the lead and the electrode, the impedance of the electrode was matched to the Thevenin impedance of the lead. Although perfect matching cannot be reached with single element, imaginary part of the sum of lead Thevenin impedance, electrode impedance, and impedance of inductor can be adjusted to zero. A cylindrical copper electrode was used with a radius of $2.2 \mathrm{~mm}$ and length of $7 \mathrm{~mm}$, which are close to dimensions of a commercial electrode. During the experiments, a gel phantom with a conductivity of $0.14 \mathrm{~S} / \mathrm{m}$ and relative permittivity of 60 was used. The circuit model parameters of the cylindrical electrode were found for the medium which has the same electrical properties of the phantom used during the experiments. Bare leads with a radius of $1 \mathrm{~mm}$ and lengths 10 and $40 \mathrm{~cm}$ were used. Before the experiments, matching concept was tested using MoM simulations. The spherical electrode was connected to a bare lead with a radius of $0.1 \mathrm{~mm}$ and length of $25 \mathrm{~cm}$. An inductor was placed between the electrode and the lead. The Thevenin equivalent of the bare lead was found using the MoTLiM. The electrode model was connected to the Thevenin equivalent of the lead via a matching inductor. By changing the value of the inductance to range from 1 and $250 \mathrm{nH}$, the real part of the dissipated power was calculated for the electrode impedance using the MoTLiM. Then, E-field and SAR were calculated on the surface of the spherical electrode using MoM simulations and compared with MoTLiM simulation results.

Then, matching the lead impedance to the electrode impedance was demonstrated using experiments. A bare wire with a radius of $0.5 \mathrm{~mm}$ and length of $20 \mathrm{~cm}$ was connected to the cylindrical electrode with a radius of $2.2 \mathrm{~mm}$ and length of $7 \mathrm{~mm}$ via a custom-made matching inductor. The value of the inductance was varied from 20 to $160 \mathrm{nH}$. Lead length was chosen close to the resonance length and inductors were wound to fit inside a lead. For each inductance value, the temperature rise at the electrode was measured. For each condition, the real part of the dissipated power associated with the electrode impedance was calculated using the MoTLiM and the electrode model. These calculations were compared with the experimental data. All calculated power values were for $1 \mathrm{~V} / \mathrm{m}$ incident $\mathrm{E}$-field; however, during experiments, much higher incident field was used.

\section{RESULTS}

\section{A. Simulation results}

Circuit model parameters of the PEC box $(1.2 \times 4.4 \times 5 \mathrm{~cm})$ inside the medium with relative permittivity 55 and conductivity $0.17 \mathrm{~S} / \mathrm{m}$, at $123 \mathrm{MHz}$, which is the resonance frequency of Siemens $3 \mathrm{~T}$ TimTrio scanner under a $1 \mathrm{~V} / \mathrm{m}$ incident field were found as follows: $V_{c}=-20.5+j 11.3 \mathrm{mV}$ and $Z_{c}=0.96$ $-j 1.3 \Omega$. These values were used as boundary conditions to solve the induced currents on the leads. In Fig. 5, induced currents on the leads with lengths $40.3,35.3$, and $27.3 \mathrm{~cm}$ were solved for with MoTLiM and compared with the MoM simulations. The blue solid lines are the MoTLiM solutions, and the red dashed lines are the MoM solutions of the induced currents. At $s=0$, the leads were connected to the IPG case. The errors were $6.1 \%, 8.7 \%$, and $6.0 \%$ for the lead lengths $40.3,35.3$, and $27.3 \mathrm{~cm}$, respectively. The circuit model parameters of the spherical electrode were found inside the medium with relative permittivity 81 and conductivity $0.42 \mathrm{~S} / \mathrm{m}$, and at 

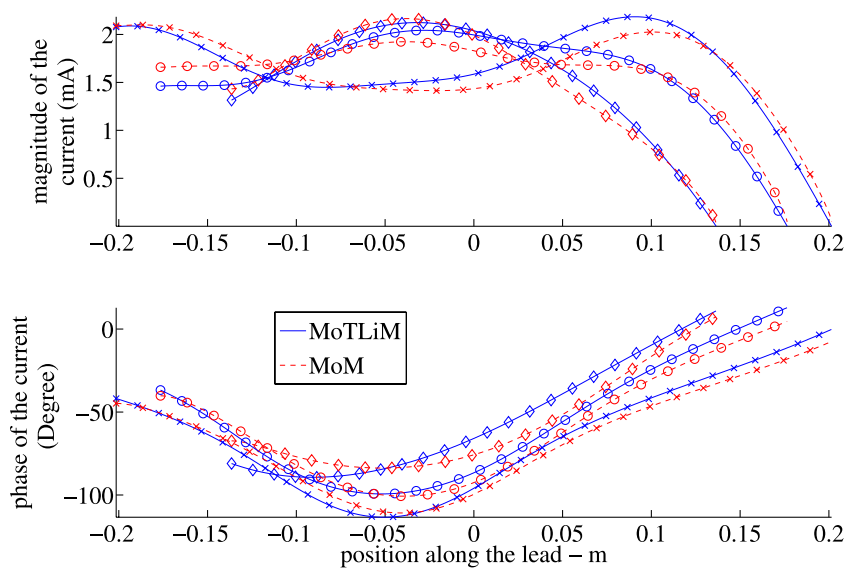

Fig. 5. The induced currents on the leads with the IPG case connection under $1 \mathrm{~V} / \mathrm{m}$ uniform E-field incidence. Three bare leads with radius $1 \mathrm{~mm}$ and lengths $\left(l_{m}\right) 40.3 \mathrm{~cm}(\times), 35.3 \mathrm{~cm}(\circ)$, and $27.3 \mathrm{~cm}(\diamond)$ were connected to a PEC IPG case at the position $s=-l_{m} / 2$. The blue solid lines are the solution obtained from the MoTLiM and IPG case model, and the red dashed lines are the MoM solution results.

$123 \mathrm{MHz}$ under $1 \mathrm{~V} / \mathrm{m}$, the incident field was found to be $V_{e}$ $=-20.5+j 4.1 \mathrm{mV}$ and $Z_{e}=149-j 137 \Omega$. Similar to the IPG case, the electrode model was used in the boundary conditions to solve for the induced currents on the leads. In Fig. 6, induced currents on the leads with lengths 25,35 , and $35 \mathrm{~cm}$ were solved for using the MoTLiM and compared with the MoM simulations. The blue solid lines are the MoTLiM solution, and the red dashed lines are the MoM solutions of the induced currents. The leads were connected to the electrodes at the ends where the position $s$ has a positive value. The errors were $4.6 \%, 4 \%$, and $3.3 \%$ for the lead lengths 25,35 , and $45 \mathrm{~cm}$, respectively.

Then, the Thevenin voltage and impedance of the bare lead with length $25 \mathrm{~cm}$ and radius $0.1 \mathrm{~mm}$ found as follows:
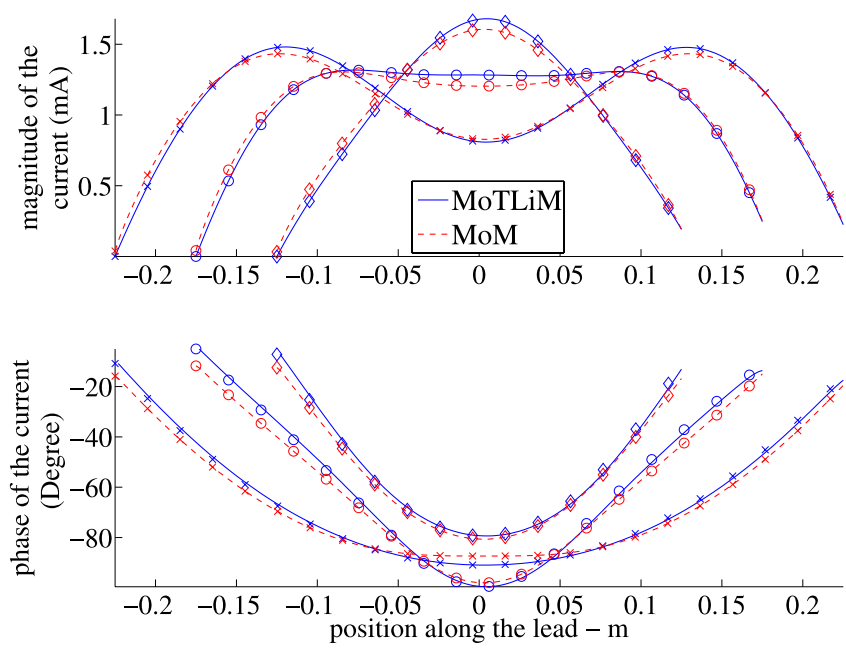

Fig. 6. The induced currents on leads with electrode connections under 1 $\mathrm{V} / \mathrm{m}$ uniform E-field incidence. The blue solid lines are the solution obtained using the MoTLiM and IPG case model, and the red dashed lines are the MoM solution results. Three bare leads with radius $0.1 \mathrm{~mm}$ and lengths $25 \mathrm{~cm}(\diamond), 35 \mathrm{~cm}(\circ)$ and $45 \mathrm{~cm}(\times)$ were connected to a spherical electrode at the end where the position in the figure has a positive value.

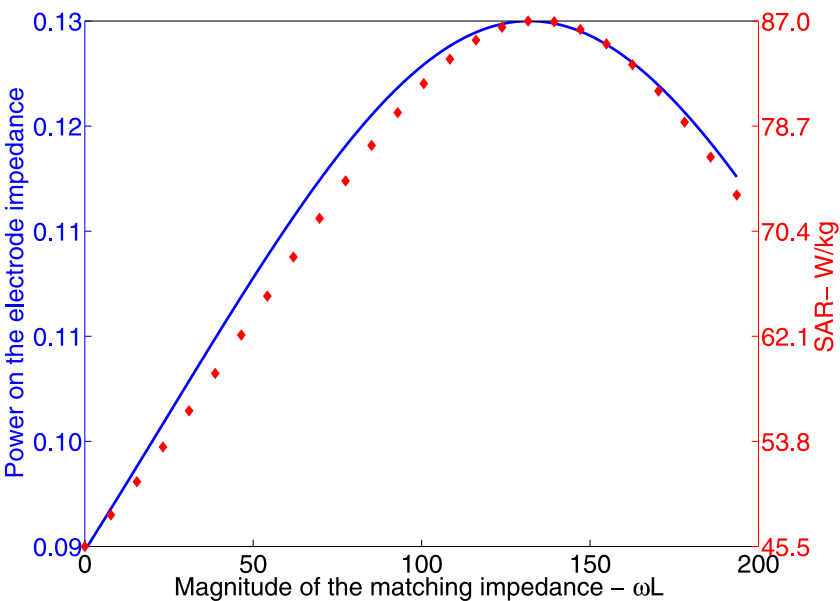

Fig. 7. The blue solid line is the real part of the dissipated power for the impedance of the spherical electrode with radius $1 \mathrm{~mm}$. The red diamonds are the unaveraged SAR at a point $0.1 \mu \mathrm{m}$ away from the electrode obtained using MoM simulations. The value of the matching inductance was changed from 1 to $250 \mathrm{nH}$.

$V_{\text {th }}=8.7-j 28.6 \mathrm{mV}$ and $Z_{\text {th }}=37.7+j 4.88 \Omega$. In Fig. 7 , the real power and SAR reach the maximum values, where the imaginary part of the sum of $Z_{\mathrm{th}}, Z_{e}$, and $Z_{m}$ equals zero.

\section{B. Experiment results}

In Fig. 8, blue cross sign, $\times$, indicates the rise in tip temperature when the lead is connected to IPG case. The blue plus sign, + , indicates the rise in tip temperature when both ends of the lead were floating inside the phantom. The red dashed line indicates the square of the voltage at the tip of the lead when it is connected to the IPG case. The red solid line indicates the square of the voltage at the lead tip when both ends of the lead are floating inside the phantom. The location of the

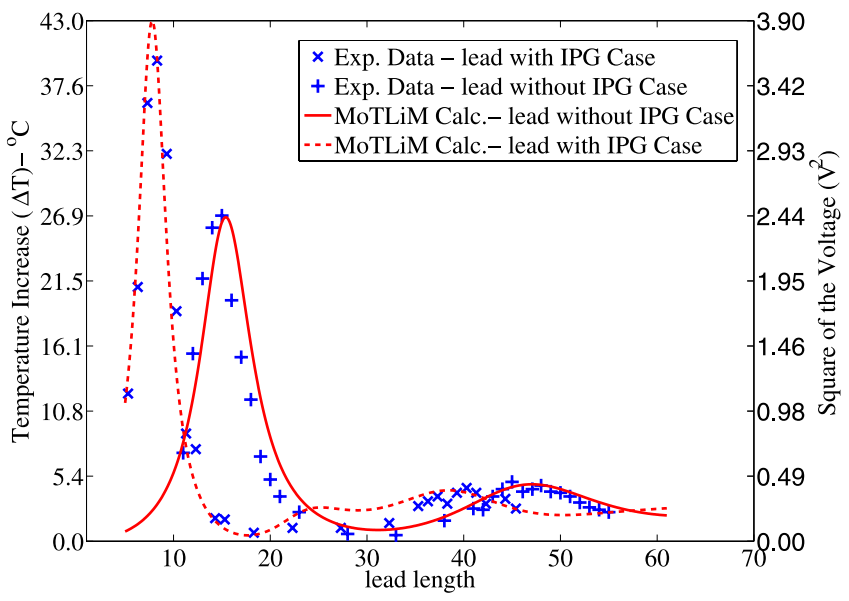

FIG. 8. The dashed red line is the square of the hypothetical voltage at the end of the lead for different lead lengths when the lead is connected to the IPG case. The solid red line is the square of the hypothetical voltage at the end of the lead for different lead lengths. Blue $\times$ is the measured temperature rise at the lead tip when the lead is connected to the IPG case. The blue + is the measured rise in tip temperature of the lead with no connection. Left $y$ axis is the temperature rise and right $y$ axis is the square of the hypothetical voltage. Axis scales are adjusted for the best visualization of the trend. 


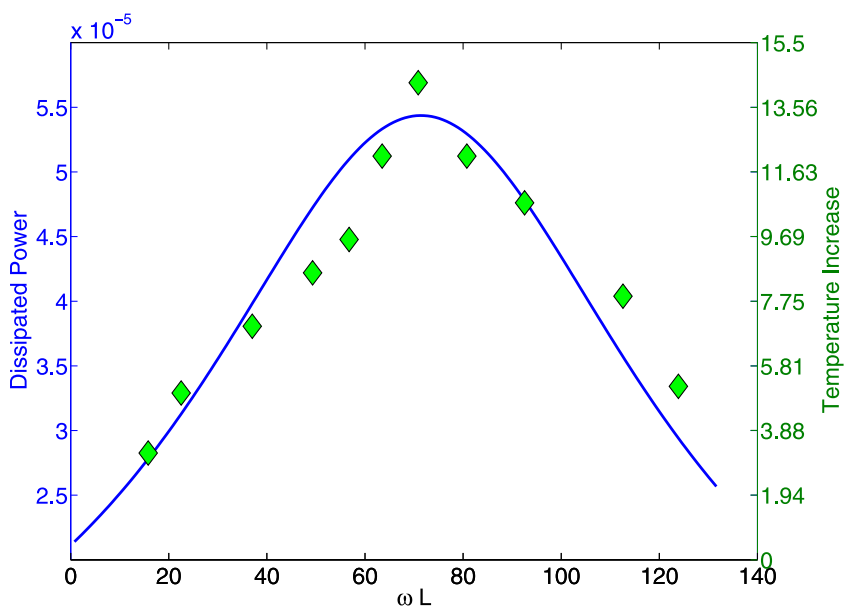

FIG. 9. The blue solid line is the calculated dissipated power for the real part of the electrode impedance with respect to the value of the matching impedance. The green diamonds indicate the measured temperature rise with respect to the value of the matching impedance at the electrode end. Right $y$ axis is the temperature rise and left $y$ axis is the square of the hypothetical voltage. Axis scales are adjusted for the best visualization of the trend.

resonance peaks is calculated with less than $13 \%$ error. The rise in tip temperature when the lead is connected to the IPG case is predicted with less than $4 \%$ error.

In Fig. 9, the green diamonds indicate the rise in tip temperature with respect to the value of the matching impedance. The blue solid line is the calculated real power dissipated for the electrode impedance. The matching of the electrode impedance to the lead Thevenin impedance is shown, and it is also predicted using MoTLiM and the electrode circuit model. The value of the matching impedance was predicted with $0.5 \%$ error.

\section{DISCUSSION AND CONCLUSION}

In this work, the electrode and case parts of an active implantable device (IPG) were modeled with an electrical circuit at the operating frequency of $3 \mathrm{~T}$ scanner, which was available on site. A novel method was developed to find the parameter values of the circuit model at RF. The electrical circuit model contains an impedance and a voltage source. The impedance models the interaction of the electrode/IPG case with the tissue, and the voltage source shows the effect of the incident field. To find the parameter values, both MoTLiM and MoM simulations were used. The MoM was used because of its capability of accurately solving currents on wires; however, any other numerical method can also be used. The values of the circuit model parameters were tested using MoM simulations and MRI experiments. A uniform E-field incidence was used for all of the derivations, simulations, and experiments for the sake of simplicity. All concepts can be rederived using the methods explained here for a known E-field incidence. It is shown that MoTLiM ${ }^{11}$ is capable of solving currents for nonuniform E-field as long as the tangential component of the E-field is known. The performance of the MoTLiM combined with the proposed circuit models of IPG case and the elec- trodes, however, has not been tested under nonuniform electric field. If the field variation is small compared to electrode and IPG case size, we predict that the overall performance of the proposed analysis method will be similar to that of MoTLiM alone. As it was discussed in the original MoTLiM study, ${ }^{11}$ the current distribution on the lead can be predicted reasonably well when the electric field variation along the lead is small. Also, during these analyses, bare cylindrical leads were used with a rectangular IPG case, a cylindrical electrode, and a spherical electrode. In this study, the effect of IPG case and electrode geometry on the induced currents has not been analyzed rigorously. We predict that the induced current distribution on the leads that are connected to the different electrodes and IPG cases will have similar sinusoidal standing wave behavior. For any shape of IPG cases and electrodes, one should find the corresponding model parameters using the proposed method. Also, in this study, lead is directly connected to the IPG case. Although in some implants this connection can be done using lumped elements, in theory these lumped elements can be incorporated into proposed model. Of course, experimental verification needs to be done for such modification to our proposed model.

It is also highly possible that the AIMD lies inside different tissues. Because defined IPG case and electrode impedances are medium dependent, they must be found using the electrical properties of the medium surrounding the IPG case and electrode. Also, the lead could be passing through different tissues. For this situation, the lead can be considered as serially connected transmission lines with different $k_{t}$ and $Z$ values.

Using MoTLiM, the lead part was analyzed, and with the proposed circuit models, the effects of the IPG case, electrode, and any lumped circuit element placed between these parts could be analyzed. With this theory, a complex scattering problem was recast as a circuit problem, and any modification could be solved easily.

To test the IPG case, induced currents were calculated using the MoTLiM, and the circuit model was then compared with the MoM simulations. The error in the solutions was below $10 \%$. Then, the IPG case model was tested using the MRI experiments. The square of the hypothetical voltage was calculated at the end of the lead, with the IPG case connection at the other end. The square of the hypothetical voltage was also found for the lead without a connection to any IPG case or electrode. Then, MRI experiments were conducted for these cases. A bare lead was connected to a PEC box and replaced inside a uniform phantom. The tip heating was then measured by changing the lead length. The tip temperature rise was measured for the same lead with no case connection. These data were then compared with the calculations. The locations of the resonance peaks were predicted with and without the IPG case with a less than $13 \%$ error. The change in the magnitude of the temperature rise when the IPG case was connected to the lead was predicted with a less than $4 \%$ error.

To test the electrode, induced currents on the leads were solved for different lead lengths. The MoTLiM results were compared with the MoM simulations. The error in the induced currents was less then $5 \%$. Then, the power matching concept was demonstrated with simulations and experiments. A match- 
ing inductor was placed between the electrode and the lead, and the power dissipated for the real part of the electrode impedance was calculated by changing the inductance value. When the electrode impedance was matched to the lead Thevenin impedance, the dissipated power for the real part of the electrode impedance reached its maximum. MRI experiments were conducted for the same scenario. It is shown that at the matching condition, the temperature rise at the electrode reaches its maximum. This result is important, as replacing the inductor between lead and electrode was used for preventing a temperature rise at the electrode end. ${ }^{15}$ However, it is shown that the value of the inductance must be chosen carefully, as it can affect the tip temperature rise.

The analysis was conducted to determine the square of the hypothetical voltage. In the quasistatic region, the scattered fields decayed fast due to the loss of the medium, the square of the hypothetical voltage at the tip of the lead had a linear relationship with the SAR, and the temperature rise was calculated using linear approximations ${ }^{2,16}$ from the SAR distribution. In the case for which these assumptions fail, the SAR can be solved for using the whole hypothetical voltage distribution along the lead.

With the presented data, it is shown that IPG case and electrode can be modeled with a simple circuit model. Although all modeling was done for $3 \mathrm{~T}$, which is available on site, all derivations can be easily done for $1.5 \mathrm{~T}$ as frequency is just a parameter in $k_{t}$ and $Z .{ }^{11}$ Presented circuit models can be helpful in order to choose correct IPG case, lead, and electrode combinations. In the literature, there are studies ${ }^{7}$ which show that using a lead with different electrodes will result in different temperature rise in the tissue, and these studies were mostly based on experimental methods. After defining the electrode and lead Thevenin impedances, results of these experiments can be interpreted as mismatch between these impedances. In this work, it is shown that by matching the electrode impedance to the lead Thevenin impedance, temperature rise can be maximized. This fact can also be used adversely, and impedances can be chosen as the current flowing through the electrode is small such that it will not cause excessive heating.

Also, a recent study ${ }^{17}$ shows the effect of abandoned leads on the heating of a MR conditional pacemaker system. In this case, a new MR conditional lead is placed adjacent to an old lead which is no more connected to an IPG. For analysis of the tip heating of two adjacent leads, coupling between each other must be considered. Despite the coupling between two adjacent leads, induced currents on them will still have oscillatory standing wave behavior, so the models proposed in this paper may be used for analysis of two adjacent leads. However, the model parameters must be found by considering the coupling between two leads.

In the IPG case experiments, it is shown that the presence of the IPG case drastically changes the resonance length and the amount of temperature rise. Langman et al. showed that for different lead lengths connecting IPG to the lead can either increase or decrease the tip heating. ${ }^{18}$ Here, this effect is explained with the shift of length-temperature rise curve in the presence of the IPG case. It is shown that the effect of IPG case on the tip temperature rise can be predicted using the proposed lumped element models. With the IPG case circuit model, this length can be predicted with less than $13 \%$ error. So, this fact can be useful for avoiding resonance.

Also, with the recent advancements, ${ }^{5}$ obtaining MR conditional labeling is a hot topic for AIMD producers. In determining the conditions that implanted AIMDs may be used in the MRI, worst case analysis is necessary. Currently, this process is carried out with extensive number of experiments. Using the proposed model of AIMD, the process of finding worst case conditions is simplified. As we show, the heating can be predicted using analytical formulations, once an AIMD is modeled with six parameters, a voltage source and impedance for the IPG case and the electrode, wavenumber, and impedance per length for the lead. In addition, effect of modifications on the lead can be examined. Weiss et al. ${ }^{19,20}$ proposed MRI safe transmission line using transformers and matching networks to connect transmission line sections. Their design can be easily analyzed with MoTLiM models. Inductance, resistance, and stray capacitance of transformer sections and matching networks can easily be integrated into MoTLiM and can be analyzed. Optimization of the device can easily be done with MoTLiM modeling. Ladd and Quick ${ }^{21}$ proposed placing RF chokes on the leads. Effect of these chokes can also be analyzed with MoTLiM modeling. Resistance of these chokes can be integrated on the MoTLiM model, and interaction with electrodes and IPG cases can be analyzed.

In sum, using the presented lumped circuit models of implant electrode and the case together with the lead model, MoTLiM, the effect of the IPG case and the electrode on the tissue heating can be predicted. Using proposed lumped element models together with MoTLiM, worst case condition for tissue heating can be predicted and MRI safety tests of AIMDs can be designed. Also, these models may be helpful for designing of MRI compatible AIMDs.

\footnotetext{
a) Author to whom correspondence should be addressed. Electronic mail: vacik@ee.bilkent.edu.tr

${ }^{\text {b) }}{ }_{\text {Ww. mrcomp.com. }}$

${ }^{1} \mathrm{~V}$. Acikel and E. Atalar, "Intravascular magnetic resonance imaging (MRI)," in Biomedical Imaging: Applications and Advances, edited by M. Peter (Woodhead Publishing, Cambridge, UK, 2014), pp. 186-213.

${ }^{2}$ C. J. Yeung, R. C. Susil, and E. Atalar, "RF safety of wires in interventional MRI: Using a safety index," Magn. Reson. Med. 47(1), 187-193 (2002).

${ }^{3}$ M. K. Konings, L. W. Bartels, H. F. Smits, and C. J. Bakker, "Heating around intravascular guidewires by resonating RF waves," J. Magn. Reson. Imaging 12(1), 79-85 (2000).

${ }^{4}$ F. G. Shellock, "Radiofrequency energy-induced heating during mr procedures: A review," J. Magn. Reson. Imaging 12(1), 30-36 (2000).

${ }^{5}$ P. A. Bottomley, A. Kumar, W. A. Edelstein, J. M. Allen, and P. V. Karmarkar, "Designing passive MRI-safe implantable conducting leads with electrodes," Med. Phys. 37(7), 3828-3843 (2010).

${ }^{6}$ D. W. Carmichael, J. S. Thornton, R. Rodionov, R. Thornton, A. McEvoy, P. J. Allen, and L. Lemieux, "Safety of localizing epilepsy monitoring intracranial electroencephalograph electrodes using MRI: Radiofrequencyinduced heating," J. Magn. Reson. Imaging 28(5), 1233-1244 (2008).

${ }^{7}$ P. Nordbeck et al., "Reducing RF-related heating of cardiac pacemaker leads in MRI: Implementation and experimental verification of practical design changes," Magn. Reson. Med. 68(6), 1963-1972 (2012).

${ }^{8}$ W. R. Nitz, A. Oppelt, W. Renz, C. Manke, M. Lenhart, and J. Link, "On the heating of linear conductive structures as guide wires and catheters in interventional MRI,” J. Magn. Reson. Imaging 13(1), 105-114 (2001).
} 
${ }^{9}$ K. B. Baker, J. A. Tkach, J. A. Nyenhuis, M. Phillips, F. G. Shellock, J. Gonzalez-Martinez, and A. R. Rezai, "Evaluation of specific absorption rate as a dosimeter of mri-related implant heating," J. Magn. Reson. Imaging 20(2), 315-320 (2004).

${ }^{10}$ A. Roguin, M. M. Zviman, G. R. Meininger, E. R. Rodrigues, T. M. Dickfeld, D. A. Bluemke, A. Lardo, R. D. Berger, H. Calkins, and H. R. Halperin, "Modern pacemaker and implantable cardioverter/defibrillator systems can be magnetic resonance imaging safe in vitro and in vivo assessment of safety and function at 1.5 T," Circulation 110(5), 475-482 (2004).

${ }^{11} \mathrm{~V}$. Acikel and E. Atalar, "Modeling of radio-frequency induced currents on lead wires during MR imaging using a modified transmission line method," Med. Phys. 38(12), 6623-6632 (2011).

${ }^{12} \mathrm{D}$. Zemann and K. Moertlbauer, "Using transmission line theory to analyze RF induced tissue heating at implant lead tips," Proceedings of International Society for Magnetic Resonance in Medicine (2012).

${ }^{13} \mathrm{C}$. J. Yeung and E. Atalar, "A green's function approach to local RF heating in interventional MRI,” Med. Phys. 28(5), 826-832 (2001).

${ }^{14}$ S. Gabriel, R. Lau, and C. Gabriel, "The dielectric properties of biological tissues: II. Measurements in the frequency range $10 \mathrm{~Hz}$ to $20 \mathrm{GHz}$,' Phys. Med. Biol. 41(11), 2251-2269 (1996).
${ }^{15}$ R. C. Susil, C. J. Yeung, H. R. Halperin, A. C. Lardo, and E. Atalar, "Multifunctional interventional devices for MRI: A combined electrophysiology/MRI catheter," Magn. Reson. Med. 47(3), 594-600 (2002).

${ }^{16}$ D. Shrivastava and J. T. Vaughan, "A generic bioheat transfer thermal model for a perfused tissue,” J. Biomech. Eng. 131(7), 074506 (2009).

${ }^{17}$ E. Mattei, G. Gentili, F. Censi, M. Triventi, and G. Calcagnini, "Impact of capped and uncapped abandoned leads on the heating of an mr-conditional pacemaker implant,” Magn. Reson. Med. 73(1), 390-400 (2015).

${ }^{18}$ D. A. Langman, I. B. Goldberg, J. P. Finn, and D. B. Ennis, "Pacemaker lead tip heating in abandoned and pacemaker-attached leads at 1.5 tesla mri," J. Magn. Reson. Imaging 33(2), 426-431 (2011).

${ }^{19}$ S. Weiss, P. Vernickel, T. Schaeffter, V. Schulz, and B. Gleich, “Transmission line for improved RF safety of interventional devices," Magn. Reson. Med. 54(1), 182-189 (2005).

${ }^{20} \mathrm{P}$. Vernickel, V. Schulz, S. Weiss, and B. Gleich, "A safe transmission line for MRI,” IEEE Trans. Biomed. Eng. 52(6), 1094-1102 (2005).

${ }^{21}$ M. E. Ladd and H. H. Quick, "Reduction of resonant RF heating in intravascular catheters using coaxial chokes," Magn. Reson. Med. 43(4), 615-619 (2000). 